\title{
S IIAНАЛИТИЧЕСКАЯ РАБОТА
}

А.C. Абраамян

\section{МЕТОДЫ ВЫЯВЛЕНИЯ РИСКОВ ВЕНЧУРНОГО ИНВЕСТИРОВАНИЯ ПРЕДПРИНИМАТЕЛЬСКИХ СТРУКТУР}

Аннотация: Рассмотрены экономико-математические методы выявления рисков; определены критерии для учета рисков венчурного инвестирования предпринимательских структур; исследованы ограничения использования венчурного капитала для финансирования инновационных проектов.

Методология выявления рисков венчурного инвестирования предпринимательских структур базируется на применении экономико-математических методов, которые можно разделить на методы решения прямых и обратных задач, а также методы исследования чувствительности.

Ключевые слова: Экономика, предпринимательство , риски, венчур, структуры, методы, инвестирование, исследования, задачи, чувствительность

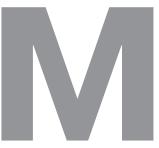

етодология выявления рисков венчурного инвестирования предпринимательских структур базируется на применении экономико-математических методов, которые можно разделить на методы решения прямых и обратных задач, а также методы исследования чувствительности.

В прямых задачах оценка риска и определение его уровня происходят на основе заранее полученной, известной информации. В обратных задачах априорно задаются ограничения на один или несколько варьируемых параметров и постулируется цель соблюсти эти ограничения при приемлемом (также заранее установленном) уровне риска.

Метод исследования чувствительности становится особо важным инструментом при недостаточной точности исходной информации. Основополагающая идея метода состоит в анализе того, в какой степени из- меняются результативные (критериальные) показатели при варьировании параметров модели. При этом могут анализироваться распределения вероятностей величин, математические ожидания, дисперсии и другие статистические оценки. Выводы из анализа чувствительности проекта обязательно отражают степень достоверности и практической применимости результатов, полученных при таком риск-анализе.

В случае признания результатов анализа неудовлетворительными аналитик вынужден прибегнуть к перечисленным ниже вариантам:

- изменить методики обработки данных с целью уменьшения чувствительности отклика;

- перестроить математическую модель анализа предпринимательских рисков;

- отказаться от проведения количественного анализа предпринимательских рисков. 
В наибольшей степени применимыми для анализа рисков венчурного инвестирования предпринимательских структур в настоящее время считаются следующие классы математических моделей, учитывающих неопределенность и различающиеся по способам ее описания:

- стохастические модели;

- лингвистические модели;

- $\quad$ нестохастические (игровые) модели ${ }^{1}$.

Всё многообразие существующих на сегодня методов оценки риска, а также связанных с ними теоретических и эмпирических моделей, можно классифицировать по следующим 3 направлениям.

1. Первый классификационный признак использование вероятностных распределений - приводит к группировке методов, не учитывающих распределения вероятностей, и методов, в которых так или иначе задействованы подобные распределения.

2. Второй способ разграничения строится на различии вероятностных методов и методов выборочных, т.е. по способу учета вероятности либо реализации каждого отдельного значения исследуемого признака, либо проведения анализа с учетом функциональных форм распределения вероятностей.

3. В зависимости от способов нахождения результирующих показателей по построенной модели применяют аналитический метод или имитационный метод.

Вероятностные методы основаны на положениях теории вероятностей и предполагают, что для построения и расчетов по модели необходимо знать распределения вероятностей анализируемых переменных. Выборочные методы также используют статистические подходы, но базируются на проведении рас-

\footnotetext{
${ }^{1}$ Гориков А.Ф., Евтеев Б.В., Кориунов В.А. Компьютерное моделирование менеджмента. - М.: Экзамен, 2007. c. $20-21$.
}

четов по выборкам данных из генеральной совокупности и получении соответствующих выборочных характеристик.

Целевой подход в методах моделирования принятия решений означает, что при конструировании модели должны быть четко заданы цели ее функционирования. Переформулирование целей ведет к перестройке, реконструкции самой модели и выполнению новых численных расчетов. Понятно, что это потребует дополнительных затрат, а потому, в большинстве случаев, лицо принимающее решение, старается не изменять целевые показатели и тем самым не прибегать к реконструкции модели. Применение целевого подхода целесообразно, если решения принимаются в однотипных, аналогичных ситуациях, а цели всякий раз точно заданы ${ }^{2}$.

Системный подход при принятии решений ассоциируется с построением модели, максимально адекватно отражающей реальность. Таким образом построение модели происходит не исходя из заранее сформулированной стройной системы целей, а ориентируясь на ключевой при анализе аспект реальности. В результате расчетов по модели и ее оценки получается скорее не оптимальная стратегия действий, а подробное описание поведения реальной системы. Система целей выбирается апостериорно, а решение принимается на основе прогнозной информации о поведении системы.

При аналитическом способе быстрое нахождение решения и получение результатов достигается непосредственным использованием экзогенных переменных. Недостатком метода признана необходимость упрощения поставленной задачи для того, чтобы достичь соответствия с требованиями применяемого математического аппарата. Кроме того, воз-

\footnotetext{
${ }^{2}$ Методические рекомендации по разработке региональных программ поддержки малого бизнеса. - М.: Институт бизнеса. - 2003. - с.18.
} 


\section{Тренды и управление - №1(1) • 2013}

никают проблемы с интерпретируемостью полученных выводов, т.е. метод оказывается «непрозрачным» в плане обоснования предлагаемого решения.

Имитационный способ предполагает обращение к алгоритмам пошагового приближения к искомому результирующему показателю. К преимуществам такого инструментария относят очевидность всех расчетов, простоту восприятия всеми участниками процесса планирования полученных результатов анализа. Из недостатков метода следует упомянуть большие вычислительные затраты и, иногда, большой объем выходной информации ${ }^{3}$.

Учет рисков венчурного инвестирования предпринимательских структур в перспективном планировании должен проводиться с целью выявления наиболее вероятных и серьезных рисков, а также для разработки мероприятий по их снижению или компенсированию.

На степень рисков венчурного инвестирования предпринимательских структур непосредственным образом влияют как факторы внешней среды, так и внутренние условия предприятий, при этом можно утверждать о наличии так называемого обратного синергетического эффекта, когда общее увеличение рисков венчурного инвестирования предпринимательских структур вызвано сочетанием факторов внутреннего и внешнего характера.

Критериями для учета рисков венчурного инвестирования предпринимательских структур могут выступать экономические, информационно-технологические, организационные и страновые критерии, которые объединяют различные виды рисков. Данные критерии и виды рисков должны быть учтены в рамках создания бизнес-концепции предпринимательской структуры, причем относятся как к стадии создания данной структуры, так и к

\footnotetext{
${ }^{3}$ Лычкина Н.Н. Имитационное моделирование экономических процессов. - М.: ГУУ. - 2005. - с. 11.
}

этапу выработки мер для оптимизации работы уже функционирующей структуры ${ }^{4}$.

Следует более подробно остановиться на вопросе, связанном с созданием бизнесконцепции или бизнес-плана предпринимательской структуры, наличие которого в подавляющем большинстве случаев необходимо для принятия положительного решения о вложении венчурных инвестиций. В рамках диссертационного исследования был проведен анкетный опрос среди руководителей 60 российских компаний из трех регионов Федерации, работающих в сфере малого инновационного предпринимательства.

Организация опроса подразумевала, что на каждый вопрос анкетируемый должен был выбрать единственный, самый важный для него ответ из фиксированного перечня предложенных вариантов. Помимо этого не допускался отказ от ответа, при затруднениях с выбором рекомендовалось остановиться на наиболее приемлемом варианте.

В таблице 1 сведены данные ответов на вопрос «В чем состоят ограничения для использования венчурного капитала в Вашей фирме при финансировании инновационных проектов?».

Треть респондентов в качестве ограничений отмечают общую неразвитость венчурного бизнеса в России. Эта проблема стоит одинаково остро во всех рассмотренных в рамках данного анкетирования регионах.

Следующей по значимости (21,7\% опрошенных) идёт необходимость составления подробного и обоснованного бизнес-плана инновационного проекта.

Еще 10 лет назад среди основных факторов, препятствующих инновационной деятельности в Пермском крае, выделялось

\footnotetext{
${ }^{4}$ Керимов Р.Ф. Применение системы сбалансированных показателей для выявления предпринимательских рисков в гостиничном бизнесе // Экономика. Налоги. Право. 2011 г.- № 3
} 
Аналитическая работа

то обстоятельство, что при наличии перспективных идей, основная их масса не проработана и не оформлена в реалистичные бизнес-планы ${ }^{5}$.

В настоящее время данная проблема остается второй по значимости для всех обследованных предприятий.

Бизнес-концепция представляет собой краткое изложение ведущего замысла бизнес-деятельности и включает в себя:

- состав и содержание основных бизнес-процессов производства и реализации потребителю конечных продуктов;

Таблииа 1 Ограничения для использования венчурного капитала для финансирования инновачионных проектов

\begin{tabular}{|c|c|c|c|c|}
\hline \multirow[t]{2}{*}{ Ограничение } & \multicolumn{4}{|c|}{$\begin{array}{l}\text { Количество и доля (в скобках) респондентов, } \\
\text { указавших данное ограничение как основное }\end{array}$} \\
\hline & г. Москва & $\begin{array}{c}\text { Пермский } \\
\text { край }\end{array}$ & $\begin{array}{c}\text { Нижегородская } \\
\text { область }\end{array}$ & Итого \\
\hline $\begin{array}{l}\text { Необходимость составления под- } \\
\text { робного бизнес-плана проекта }\end{array}$ & $\begin{array}{c}6 \\
(28,6 \%)\end{array}$ & $\begin{array}{c}5 \\
(20,8 \%)\end{array}$ & $\begin{array}{c}2 \\
(13,3 \%)\end{array}$ & $\begin{array}{c}13 \\
(21,7 \%)\end{array}$ \\
\hline $\begin{array}{l}\text { Отсутствие информации о вен- } \\
\text { чурных инвестициях в нашем } \\
\text { регионе }\end{array}$ & $\begin{array}{c}3 \\
(14,3 \%)\end{array}$ & $\begin{array}{c}3 \\
(12,5 \%)\end{array}$ & $\begin{array}{c}5 \\
(33,3 \%)\end{array}$ & $\begin{array}{c}11 \\
(18,3 \%)\end{array}$ \\
\hline $\begin{array}{l}\text { Неуверенность в том, что проект } \\
\text { будет соответствовать требовани- } \\
\text { ям венчурного инвестора }\end{array}$ & $\begin{array}{c}2 \\
(9,5 \%)\end{array}$ & $\begin{array}{c}4 \\
(16,7 \%)\end{array}$ & $\begin{array}{c}1 \\
(6,7 \%)\end{array}$ & $\begin{array}{c}7 \\
(11,7 \%)\end{array}$ \\
\hline $\begin{array}{l}\text { Неразвитость данного вида фи- } \\
\text { нансирования }\end{array}$ & $\begin{array}{c}7 \\
(33,3 \%)\end{array}$ & $\begin{array}{c}8 \\
(33,3 \%)\end{array}$ & $\begin{array}{c}5 \\
(33,3 \%)\end{array}$ & $\begin{array}{c}20 \\
(33,3 \%)\end{array}$ \\
\hline $\begin{array}{l}\text { Отсутствие четких критериев } \\
\text { выхода венчурного бизнеса из } \\
\text { компании }\end{array}$ & $\begin{array}{c}3 \\
(14,3 \%)\end{array}$ & $\begin{array}{c}4 \\
(16,7 \%)\end{array}$ & $\begin{array}{c}2 \\
(13,3 \%)\end{array}$ & $\begin{array}{c}9 \\
(15,0 \%)\end{array}$ \\
\hline Итого & $\begin{array}{c}21 \\
(100 \%)\end{array}$ & $\begin{array}{c}24 \\
(100 \%)\end{array}$ & $\begin{array}{c}15 \\
(100 \%)\end{array}$ & $\begin{array}{c}60 \\
(100 \%)\end{array}$ \\
\hline
\end{tabular}

Эта сложность в несколько большей степени волнует представителей компаний г. Москвы, а наименее характерна для организаций Нижегородской области.

\footnotetext{
${ }^{5}$ Бернштам Е. С., Кузнецов А. В. Факторы и показатели инвестиционной привлекательности российских регионов // Федеративные отношения и региональная социально-экономическая политика. - 2002. - № 10. - с. 3-12.
}

- основные особенности деятельности предприятия, определяющие его конкурентоспособность в изменяющейся бизнес-среде;

- перечисление бизнес-рисков, возникающих в хозяйственной деятельности предприятия.

Что касается конкретных количественных или качественных характеристик кри- 


\section{Тренды и управление - №1(1) • 2013}

териев для учета рисков венчурного инвестирования предпринимательских структур в перспективном планировании, то их состав и пороговые значения необходимо обосновывать особо для различных объектов предпринимательской деятельности.

Общий алгоритм управления рисками венчурного инвестирования предпринимательских структур в перспективном планировании может быть представлен следующим образом. На первом этапе происходит выявление и оценка конкретного вида предпринимательского риска. В зависимости от результатов этапа предлагаются методы воздействия на риск с обязательным прогнозом эффективности каждого метода. Далее следует окончательный выбор метода воздействия на риск. Заключительным этапом алгоритма является апостериорный контроль результатов, в качестве которых обычно рассматриваются снижение или невозрастание риска.

Рассматривая методы управления рисками венчурного бизнеса, следует отдельно выделить рисковый аспект, который позволяет перечислить ключевые виды риска для финансируемого проекта, выделить те из них, которые определяются внутренней или внешней средой инвестируемой компании, а также сферой приложения проекта и степенью его коммерциализации 6 .

Решение проблемы управления рисками подразумевает, что каждый инновационный проект еще до включения его в программу венчурного финансирования должен быть всесторонне проанализирован с позиции поиска слабых мест, оценки вероятности неудачной реализации и т.п. Такой анализ позволяет наде-

\footnotetext{
${ }^{6}$ Керимов Р.Ф. Влияние факторов внешней и внутренней среды на предпринимательские риски гостиничного бизнеса // Сборник материалов международной научнопрактической конференции «Национальные интересы РФ и финансовое оздоровление экономики»-М.: ВГНА Минфина РФ, - 2010.
}

яться на принятие адекватных мер по ограничению и нивелированию выявленных рисков. В ходе реализации инновационного проекта венчурный инвестор, участвующий в данном проекте, с помощью тех или иных организационных структур должен осуществлять мониторинг состояния и хода выполнения проекта с тем, чтобы иметь возможность своевременно обнаружить новые фактора риска и выработать комплекс необходимых мер.

Серьезность инвестиционного риска можно оценить в количественной форме, например, оценив вероятность неполучения дохода и/ или потери инвестиций. Также целесообразно провести декомпозицию инвестиционного риска на составляющие и, в том числе, оценить значимость следующих видов риска:

- законодательного (правовые регуляторы инвестирования в конкретную отрасль, формальный порядок использования ресурсов);

- общеполитического (возможная нестабильность на уровне государства и отдельного региона);

- макроэкономического (кризисные явления и тенденции);

- финансового (сбалансированность инвестиционного портфеля);

- социального (уровень социальной напряженности);

- экологического (загрязнение окружающей среды) и др.

Представим основные соображения по ограничению негативного влияния перечисленных и ряда других типов рисков.

Законодательные риски по сути сводятся к вероятности кардинального изменения применимой нормативно-правовой базы во время реализации проекта. О предотвращении таких изменений говорить некорректно, но определенного рода подготовка к таким неблагоприятным внешним событиям может быть проведена при условии постоянного 
высококвалифицированного юридического сопровождения инновационного проекта. Дополнительную уверенность инвесторам придают государственные гарантии российского правительства.

Категория общеполитических рисков также включает события, традиционно относимые к форс-мажорным - это риски, обусловленные вероятностью начала военных действий, гражданских беспорядков и т.п. Примыкают к ним и риски возникновения чрезвычайных ситуаций природного и техногенного характера, связанные с авариями, катастрофами, стихийными бедствиями. Очевидно, что ограничение таких рисков относится к сфере общегосударственной политики, а потому их обсуждение явно выходит из контекста настоящего диссертационного исследования.

Ограничение чисто финансовых рисков достигается непосредственным участием в проекте венчурного инвестора, обеспечением с его стороны контроля деловой активности в рамках проекта. Возможно также привлечение страховой компании, специализирующейся на страховании рисков подобного типа.

Понятие «рыночный риск» включает риск трудностей сбыта инновационной продукции и, как следствие, риск неполучения доходов, ожидающихся венчурным инвестором. Минимизация подобного риска возможна путем использования на этапе разработки проекта результатов актуальных маркетинговых исследований, а на этапах реализации маркетинговым сопровождением проекта. Предполагается при этом, что будет выполняться анализ динамики спроса и предложения, при необходимости для адаптации под постоянно меняющиеся рыночные условия будет производиться корректировка ценовой, сбытовой, рекламной стратегии проекта.

К числу стандартных инвестиционных рисков относят и монопольный риск. Обычно под ним понимают зависимость проекта от ограниченного числа крупных клиентов. Изменение их планов или платежеспособности может приводить к серьезным последствиям с точки зрения успешности инновации. Этот риск нивелируется усилиями по поиску дополнительных клиентов, активной маркетинговой программой.

Технические риски минимизируются за счет реализации конкурсных механизмов привлечения к проекту соисполнителей, поставщиков, подрядчиков.

К группе региональных рисков следует отнести риск несоответствия проекта ресурсным возможностям региона и риск несоответствия проекта текущему состоянию инфраструктуры региона. Понятие инфраструктуры может истолковываться в расширительном смысле, включая в него не только коммуникации, но и наличие инвестиционных институтов, качество банковского обслуживания и т.п. Оба этих вида риска должны быть устранены путем анализа доступных ресурсов на этапе разработки инновационного проекта.

Социальные риски должны оцениваться на базе анализа макроэкономических показателей регионального уровня. Основными при этом могут выступать характеристика доходов населения, уровень занятости и наличие квалифицированных трудовых ресурсов.

Многие риски после их детального изучения могут быть объединены и перегруппированы, что позволит свести весь спектр рисковых ситуаций к компактному набору рисков, классифицированных по определенным признакам, важным для рассматриваемого проекта. В идеале, если такая возможность может быть реализована, изучением рисков инновационной деятельности должно заниматься специализированное аналитическое подразделение или организация.

Систематизировав возникающие риски, например, на региональном уровне, можно подойти к решению проблемы страхования 


\section{Тренды и управление - №1(1) • 2013}

венчурных инвестиций, в разработке которой, конечно, должны принять участие страховые фирмы и финансовые организации.

\section{Библиография:}

1. Горшков А.Ф., Евтеев Б.В., Коршунов В.А. Компьютерное моделирование менеджмента. - М.: Экзамен, 2007. - с.20-21.

2. Методические рекомендации по разработке региональных программ поддержки малого бизнеса. - М.: Институт бизнеса. - 2003. - c.18.

3. Лычкина Н.Н. Имитационное моделирование экономических процессов. - М.: ГУУ. - 2005. - c. 11.

4. Миндлин Ю.Б. Стратегии инновационного развития экономики региона // Национальная безопасность/ Nota bene 2011г. -№5

5. Керимов Р.Ф. Применение системы сбалансированных показателей для выявления предпринимательских рисков в гостиничном бизнесе // Экономика. Налоги. Право. - 2011 г.- № 3.

6. Абраамян А.С. Методы выявления рисков венчурного инвестирования предпринимательских структур // Современная наука: актуальные проблемы теории и практики. Серия «Экономика и Право». № 4. 2011 г.

7. Бернштам Е. С., Кузнецов А. В. Факторы и показатели инвестиционной привлекательности российских регионов // Федеративные отношения и региональная социально-экономическая политика. 2002. - № 10. - c. 3-12.

8. Керимов Р.Ф. Влияние факторов внешней и внутренней среды на предпринимательские риски гостиничного бизнеса
// Сборник материалов международной научно-практической конференции «Национальные интересы РФ и финансовое оздоровление экономики»-М.: ВГНА Минфина РФ, - 2010.

\section{References (transliteration):}

1. Gorshkov A.F., Evteev B.V., Korshunov V.A. Komp'yuternoe modelirovanie menedzhmenta. - M.: Ekzamen, 2007. - s.20-21.

2. .Lychkina N.N. Imitatsionnoe modelirovanie ekonomicheskikh protsessov. - M.: GUU. 2005. - s. 11.

3. Mindlin Yu.B. Strategii innovatsionnogo razvitiya ekonomiki regiona // Natsional'naya bezopasnost'/ Nota bene - 2011g. -№5

4. Kerimov R.F. Primenenie sistemy sbalansirovannykh pokazateley dlya vyyavleniya predprinimatel'skikh riskov v gostinichnom biznese // Ekonomika. Nalogi. Pravo. - 2011 g.- № 3 .

5. Abraamyan A.S. Metody vyyavleniya riskov venchurnogo investirovaniya predprinimatel'skikh struktur // Sovremennaya nauka: aktual'nye problemy teorii i praktiki. Seriya «Ekonomika i Pravo». № 4. 2011 g.

6. Bernshtam E. S., Kuznetsov A. V. Faktory i pokazateli investitsionnoy privlekatel'nosti rossiyskikh regionov // Federativnye otnosheniya i regional'naya sotsial'no-ekonomicheskaya politika. - 2002. - № 10. - s. 3-12.

7. Kerimov R.F. Vliyanie faktorov vneshney i vnutrenney sredy na predprinimatel'skie riski gostinichnogo biznesa // Sbornik materialov mezhdunarodnoy nauchno-prakticheskoy konferentsii «Natsional'nye interesy RF i finansovoe ozdorovlenie ekonomiki» - M.: VGNA Minfina RF, - 2010. 